\title{
Editorial
}

\section{How do we know that the Antarctic environment is fine?}

$\mathrm{O}$ ne might imagine that the only continent in the world devoted to peace and science would be certain to play a leadership role in global questions that needed a scientific answer. Indeed, to a dispassionate observer, the present situation with respect to reporting on the state of the Antarctic environment must seem bizarre. All the Antarctic Treaty Consultative Parties are members of the United Nations Environment Programme, and all are apparently committed to providing State of the Environment Reports for their respective regions. Why then have some of these very nations been so reluctant to accept that Antarctica is a key part of this world and, to provide the holistic view required, needs to be included in the reporting? The list of excuses for delay is lengthy: no money; no secretariat to organize through; likely to be too expensive; no clear reason to undertake it; not needed at present; who would be the audience; how would we maintain political oversight; etc. but none of them ever appeared insoluble. This has been clearly demonstrated by the recent publication by New Zealand of a State of the Environment Report for the Ross Sea Region.

The report shows just what can be achieved with a little money, some political determination and the enthusiastic support of the science community. The report is well-researched, attractively presented and clearly argued, a real credit to Antarctica New Zealand and the New Zealand Government. The report identifies a range of gaps in our knowledge, and tabulates a list of potential indicators for future state of the environment reporting in the Ross Sea region. One of the most interesting features of this is how few long-term records there are of what they conclude are important processes or states. Equally important is the indication that many potentially valuable data are either not collected systematically or are not easily available to assess the extent and importance of human pressures at a regional level. The five key challenges listed at the end are no great surprise: significant gaps in our knowledge, lack of a regionally based approach to environmental management, inadequate management of key pressures such as tourism and fishing, identification and management of protected areas, and a lack of any agreed process for monitoring and reporting on the state of the environment. Reasonable questions arising from these might now be: how should these challenges be divided; are they all equally important and, if not, why not; and what new mechanisms are needed to address them?

The State of the European Arctic Environment was published in 1996. SCAR had begun arguing before then that Article 12 of the Protocol could best be met by state of the Antarctic environment reporting and that nations signed up to Agenda 21 from the 1992 Rio Earth Summit were obliged to support and undertake this for the Antarctic. Why then is progress so slow? How is it that a country of only four million people and a limited GDP can provide a report when those with much larger populations and very much greater resources are still arguing about how it might be done? I am sure that, at the Warsaw ATCM, New Zealand will provide a detailed costing for its exercise that should answer those still wishing to put forward financial arguments against progress. For the past six years the ATCM has been unable to agree to the different sort of initiative needed to address this problem. Perhaps now, with a key part of the solution already on the table, agreement on the continent-wide synthesis might prove to be a practicable proposition?

D.W.H. WALTON 